\title{
Emoción expresada y variables contextuales en padres de esquizofrénicos
}

RESUMEN: Se relaciona la emoción expresada (EE) de los familiares de esquizofrénicos con algunas variables sociodemográficas, estrés y apoyo social. PALABRAS CLAVE: Emoción Expresada, Apoyo Social, Estrés, Variables Sociodemográficas.

\section{Introducción}

La emoción expresada (EE) de las familias de los pacientes es un predictor de re-caída de primera magnitud en esquizofrénicos (1-4) y en otras patologías como la depresión $(5,6)$. Estudios transculturales han confirmado la asociación de una alta EE con la recaída en pacientes esquizofrénicos (7-17). Bebbington y Kuipers (18) revisaron 25 estudios sobre EE como factor pronóstico de recaída en esquizofrénicos y encontraron que recaían el $50 \%$ de los pacientes con familias altas en EE frente al $21 \%$ en bajas. Las intervenciones familiares orientadas a reducir el nivel de EE de las familias han conseguido, en muchos casos, hacer que las familias pasen a ser bajas en EE y que se reduzca la tasa de recaídas (18-33). Estas intervenciones han estado dirigidas a incidir en las actitudes de los familiares que determinan la alta EE (criticismo: 6 ó más comentarios críticos; implicación emocional excesiva: una puntuación de 3 ó más, y presencia de hostilidad) y a factores sociales presumiblemente asociados a dichas actitudes.
SUMMARY: The Expressed Emotion (EE) of relatives is compared whit some sociodemographic variables, stress and social support.

KEY WORDS: Expressed Emotion, social support, stress, sociodemographic variables.

Otro aspecto importante de cara a realizar este tipo de intervenciones familiares se refiere a la validez transcultural de este constructo. Pasaremos a revisar los estudios sobre este tema hasta la fecha. Las variaciones en las puntuaciones de las escalas de EE señalan ciertas diferencias culturales. Así, en los estudios británicos (1-4), el 52\% de las familias evaluadas fueron valoradas como bajas en $\mathrm{EE}$, frente a un tercio de las californianas (6). Aunque el número de $\mathrm{CC}$ era similar en ambas muestras, sólo un $4 \%$ de las familias americanas no hacían ningún $\mathrm{CC}$ frente al $33 \%$ observado en las familias británicas. La hostilidad fue, asimismo, más frecuente en la muestra americana ( $28 \%$ vs. $8 \%$ ), observándose cómo la presencia de $\mathrm{H}$, al igual que en la muestra de Vaughn y Leff (34), se asociaba a 6 ó más CC. No se observaron, sin embargo, diferencias transculturales en la distribución de SIE. En ambas muestras la mayoría de los padres eran bajos en SIE (punto de corte de valoración: 4 ó 5), mientras que las madres presentaron significativamente mayor puntuación en SIE que los padres. Leff y Vaugnh ( 34 bajaron el punto de corte de SIE a 3. ) 
Otros dos estudios de réplica $(7,8)$ con muestras americanas confirman el valor predictor de recaida psicótica de la EE familiar en la esquizofrenia. Confirmaron dichos trabajos las diferencias socioculturales descritas. En el estudio de Karno et al. (8) la distribución de la EE mostró un menor porcentaje de familias categorizadas como bajas en EE $(58,5 \%)$ con respecto al resto de estudios elaborados con muestra americana.

Wig et al. (35) con pacientes esquizofrénicos daneses, y Leff et al. (11) del Norte de la India, observaron que las diferencias culturales influyen en el valor predictivo de la EE. Leff et al. (11) encuentran que la EE familiar, como índice global, no es predictor de recaída en la muestra hindú, de tal forma que sólo la escala de $\mathrm{H}$ alcanzó significación estadística. Los autores atribuyen dicho hallazgo al mayor número de familias con baja EE encontradas en este medio (menor número de $\mathrm{CC}$ y menor $\mathrm{SIE}$ ).

La distribución de las puntuaciones en las escalas de EE en la muestra australiana se caracterizaba por una elevada incidencia de hogares altos en EE (74\%) y mayor similitud con las muestras americanas que con las británicas (36). Este trabajo es uno de los primeros donde las escalas de EE se asocian significativamente con variables de la estructura familiar, sociodemográficas y de historia de enfermedad.

Autores italianos como Cazullo et al. (15) y Bertrando et al. (37) nos muestran como los parámetros de EE pueden ser aplicados a la población italiana realizando, $\tan$ solo, una pequeña adaptación motivada por los factores étnicos, culturales y sociales muy diferentes de los observados en el origen del constructo. Dicha adaptación se basó en la elevación del punto de corte de SIE (de 3 a 4) lo que permitió que la tasa de readmisiones hospitalarias en pacientes de hogares altos en EE fuese significativamente mayor que la observada en los bajos en EE. Además, describen Bertrando et al. (37) que los familiares altos en calor (puntuación de 4 a 5), tanto del grupo alto en EE como del grupo bajo en dicho constructo, tienen menores tasas de recaídas. Resultado este que ya fue observado en los trabajos originales de EE. (3,34). En estudios francófonos el índice de EE tampoco ha mostrado su valor predictor de recaída usando los puntos de corte originales. $(12,38)$.

En España, Vizcarro y Arevalo (39) en una muestra de 31 pacientes (44 familiares) seleccionados en el momento del ingreso en el Hospital Psiquiátrico Provincial de Madrid aplicaron el mismo procedimiento que el empleado en los trabajos originales de $\mathrm{EE}(3,34)$, para estudiar el valor predictivo de EE en la recaída (entendida como la reaparición o exacerbación de síntomas psicóticos de tipo positivo). No hallaron diferencias significativas en la tasa de recaídas entre los hogares altos en EE y bajos en dicho constructo. La variación de los puntos de corte de las subescalas que forman el Indice de EE tampoco arrojó diferencias significativas. La toma regular de medicación y el número de ingresos previos resultaron ser mejores predictores de recaída a los 9 meses que la EE. No encontraron relación entre EE y otras variables predictoras de recidiva, excepto que los 
sujetos altos en EE tenían peor ajuste premórbido en la subescala de edad adulta de la Escala de Ajuste Premórbido de Cannon-Spoor et al. (40) que los bajos en EE.

Gutiérrez et al. (12), en 32 pacientes gallegos de medio urbano no ingresados desde como mínimo 9 meses (en remisión), encuentra que el nivel de EE es el mejor predictor aislado de recaídas. Las tasas de descompensaciones, entendidas como tiempo transcurrido desde el alta hospitalaria hasta que se evaluó la CFI y el número de recaídas (desde la administración de la CFI hasta 18 meses después) era significativamente mayor entre los pacientes de hogares altos en EE frente a los de hogares bajos en dicho constructo. Finalmente, el último trabajo publicado en el Estado Español sobre el valor predictivo de la EE (41) se refiere a 60 pacientes esquizofrénicos ingresados (por descompensación psicótica o primera crisis) en el Hospital Clínico Universitario de Valencia. El estudio de seguimiento de estos pacientes a los nueve meses y a los dos años no mostró relaciones con el índice de EE familiar (empleando los puntos de corte originales). Sin embargo, utilizando las escalas que forman el índice de EE por separado y disminuyendo el punto de corte (criticismo se baja a 4 ó más (C) encuentra que las familias altas en $\mathrm{EE}$ por criticismo, tienen pacientes con mayores tasas de recaídas psicóticas que los de familias bajas en criticismo. Las escalas de SIE y H, por su parte, no resultaban discriminativas. A los dos años de seguimiento la escala de criticismo, con el punto de corte modificado, no podía predecir, tampoco, la recaída.

Respecto a la relación de la EE con variables contextuales, Brown et al. (3) encontraron que las familias altas en EE tenían menos relaciones extrafamiliares y menos hijos viviendo en el hogar. Hammer (42), Platt (43), Goldmann (44), Fadden et al, (45) y Carpentier (46), señalan la pobreza de la red social de los familiares de esquizofrénicos. Estos autores han resaltado la carga que representa para la familia el tener un miembro con una enfermedad psiquiátrica, lo cual conlleva un aumento de ansiedad y depresión. Anderson et al. (47) describen una asociación positiva entre una pobre red social de los familiares y una enfermedad más prolongada, pero no encuentran correlación entre EE y red social. Bentsen et al. (48) encontraron que la alta SIE se asociaba a madres que viven solas con el hijo esquizofrénico y estaban más tiempo con ellos. Barrowclough et al. (49) encontraron que los padres altos en $\mathrm{CC}$ y $\mathrm{H}$ padecían depresión y más estrés personal que los bajos, encontraron también gran carga familiar y que si los familiares se atribuían la enfermedad del hijo el estrés personal era mayor.

El objetivo del presente trabajo es estudiar las características de EE en familiares de esquizofrénicos del País Vasco y su relación con las redes de apoyo familiar y el estrés.

\section{Método}

La familias que forman la muestra de esta investigación fueron extraídas al azar de los Centros de Salud Mental y Hospitales Psiquiátricos de Gipuzkoa y Bizkaia que cooperaron en el estudio. La selección de la muestra se realizó atendiendo a los siguientes criterios de selección: 
1. Familias residentes en la Comunidad Autónoma Vasca con un hijo esquizofrénico.

2. Paciente diagnosticado de esquizofrenia por un psiquiatra del propio centro, según criterios del DSM-III-R.

3. Edad del paciente entre 15 y 35 años.

4. Pacientes solteros y conviviendo con su familia de origen (padres y hermanos). 5.Las familias no debían haber recibido ningún tipo de tratamiento psicoterapéutico.

Se recogieron un total de 70 familias, en las que se evaluó a los padres y al paciente esquizofrénico y cuyas características sociodemográficas más relevantes se detallan a continuación:

La muestra ha quedado constituida por 70 familias, 64 de las cuales $(91,4 \%)$ son de medio urbano, el $65,7 \%$ de nivel económico medio y en el $64 \%$ uno o ambos padres son inmigrantes, con una media de 5,4 miembros por familia nuclear, de los cuales permanecen en el domicilio 4,5 de media. Las características más relevantes de los padres son: edad media de 58 años (s.d. 6,6, rango 44-74), el $50,7 \%$ tienen un nivel de estudios primarios completos o incompletos y el $17,4 \%$ no tienen estudios, el $54 \%$ con profesiones semi o no cualificadas y el $31 \%$ trabajadores cualificados o propietarios de pequeños negocios, el 52,9\% católicos practicantes y el $44 \%$ católicos no practicantes. Las madres, de 54 años de edad media (s.d. 6,9; rango 35-73), $60,3 \%$ tienen estudios primarios y el $23,5 \%$ sin estudios, el $73 \%$ se dedican a las labores de casa, el 70,6\% católicas practicantes y el $23,5 \%$ católicas no practicantes. De los pacientes, el $87 \%$ son varones, solo hay 9 mujeres, de 25,2 años de edad media (s.d. 4,2; rango 16-35), el $51,4 \%$ no acabaron $8^{\circ}$ de EGB, el $27 \%$ tienen FP completa o incompleta y el $15,7 \%$ superaron BUP, el $77 \%$ no tienen trabajo; en cuanto al orden entre hermanos, en el 32,9\% de los casos es el mayor, el $32,9 \%$ el segundo y el $22,9 \%$ el menor. Las características de enfermedad son: inicio 19 años de media, tipo insidioso, con una hospitalización previa de duración menor a 3 meses. El tipo de esquizofrenia predominante fue la paranoide y el curso crónico.

Variables e instrumentos de medida:

\section{1.- Emoción expresada}

Se evaluó a través de la Camberwell Family Interview (34). La EE está compuesta por 5 subescalas: Criticismo (CC), Hostilidad (H), Sobreimplicación Emocional (SIE), Comentarios Positivos (CP) y Calor (C). Las tres primeras son predictoras de recaída. El criticismo comprende los comentarios críticos (resentimiento, desaprobación o disgusto) dirigidos a conductas del paciente. Si el familiar expresa 6 ó más comentarios críticos, es calificado como alto en EE. La hostilidad está presente cuando el familiar desconfirma (rechaza como persona) al paciente. Es una escala global que puntúa de 0 a 4; si la hostilidad está presente el familiar es calificado como alto en EE. La implicación emocional excesiva es otra escala global e incluye 3 tipos de conductas: control excesivo de la conducta del paciente, respuesta emocional exagerada $y$ una conducta de sacrificio y sobreprotección excesivos. La puntuación va de 0 a 5 y se valora alto en EE a partir 
de 3. Comentarios positivos es una escala cuantitativa en la que se numeran las expresiones de valoración o aprecio. Calor es una escala global que va de 0 a 5 y valora las manifestaciones de afecto e interés.

Se midió la EE de cada padre por separado a través de la Camberwell Family Interview (CFI) (34) siendo evaluada por miembros del equipo que realizaron el curso de entrenamiento con la Dra. C. Vaughn obteniendo la fiabilidad exigida y realizando posteriormente un acuerdo interjueces obteniendo una fiabilidad de 0,82 . Teniendo en cuenta las tres primeras escalas se establece el nivel de EE (Alto/Bajo). Los puntos de corte que nosotros hemos utilizado para clasificar a los padres como altos en EE son los de Vaughn y Leff (34): $\geq 6 \mathrm{CC}$ y/o presencia de Hostilidad $y / o \geq 3$ en SIE. El índice de EE de la familia nos viene dado por el índice mayor que se obtenga en el estudio de EE de los padres, de tal forma que si uno de ellos es alto en EE, se considera a todo el hogar como alto en EE.

\section{Variables contextuales}

Como variables contextuales relacionadas con la Emoción Expresada y/o con la recaída de los pacientes esquizofrénicos hemos estudiado la red de soporte social de la familia y los acontecimientos vitales estresantes. Ambas variables combinadas permiten obtener un índice del nivel de estrés familiar y/o individual, entendido como una interacción entre el estímulo (AVE) y los factores moderadores (Apoyo social). En esta investigación se han estudiado el apoyo social de los padres y los acontecimientos vitales estresantes mediante dos cuestionarios.
APOYO SOCIAL. Para su medida se ha utilizado la Escala de Apoyo Social del Departamento de Salud Mental de la Universidad de California. Evalúa cantidad y calidad de apoyo y ha sido traducida $y$ baremada para la población española por Conde y Franch (50).

ACONTECIMIENTOS VITALES ESTRESANTES. El instrumento empleado para esta variable es la Escala de Acontecimientos Vitales del Departamento de Salud Mental de California (1981) traducida y baremada por Conde y Franch (50).

NIVEL DE ESTRES: Esta variable, como ya se ha indicado anteriormente es una combinación de las dos variables anteriores (nivel de apoyo social y acontecimientos vitales estresantes) sobre la base de los baremos establecidos por Conde y Franch (1984) para ambas escalas. Teniendo en cuenta que ambas escalas han sido administradas a los padres (padre y madre), el nivel de estrés se ha estudiado a dos niveles: individual (Padre y Madre) y familiar (ambos).

Se han categorizado dos niveles: alto riesgo de enfermar y protección ante el estrés. Para categorizar a los individuos como pertenecientes a uno de los niveles citados, se han establecido los siguientes criterios:

1. Cuando el nivel de apoyo es bajo y la suma de AVE está a nivel de moderado o alto, o cuando el apoyo es moderado y los AVE son de nivel alto. En ambos casos se ha considerado como nivel de estrés alto, es decir, el estrés al que está siendo sometido el sujeto o la familia es alto.

2. Para todos los casos con alto apoyo o apoyo moderado y AVE modera 
do o bajo, o apoyo bajo y AVE también bajo, el nivel de estrés sufrido durante el último año ha sido categorizado como bajo.

La combinación de los datos del padre y la madre en ambas variables para crear una nueva (el estrés familiar) se ha realizado mediante dos técnicas (51):

- Para la escala de AVE se ha utilizado «la suma no redundante» o puntuación maximizada, que consiste en: sumar las puntuaciones de los ítems que han sido contestados por cualquiera de los dos padres como «sí ocurrió», pero si ambos contestan igual sólo se computa la puntuación de uno de ellos. Conceptualmente la puntuación maximizada implica la asunción de que cualquier AVE que afecte a un miembro de la familia o pareja, repercute de forma más o menos directa en el resto de la familia.

-En el apoyo social se ha recurrido a la media aritmética de las puntuaciones totales de cada miembro de la pareja. Conceptualmente, si un miembro de la pareja tiene bajo apoyo social tenderá a descargar sus tensiones en el otro miembro de la pareja. Si éste otro tiene apoyo alto podrá liberarse de parte de esta carga, con lo cual la función del apoyo en esta familia quedaría compensada por uno de sus miembros. Esta «compensación», es lo que se refleja, desde un punto de vista estadístico, en la media aritmética.

\section{Resultados}

\section{1.- Relación de EE con variables sociodemográficas:}

Observamos en los resultados ob- tenidos que la EE familiar, es decir que el hogar sea alto o bajo en EE, no se relaciona con el nivel socioeconómico, ni con la movilidad social, ni con el medio urbano/ rural. Sin embargo objetivamos relación significativa con la variable inmigración $\left(\chi^{2}=6,50 ; p=0,03\right)$.

En hogares bajos en EE hay mayor número de lo esperado de la categoría que se define por un padre inmigrante. En los hogares altos en EE, sin embargo, se observa menor frecuencia. Por otra parte, cuando ambos padres son inmigrantes se observa un cierto predominio de hogares altos en EE frente a bajos en la citada variable. En el último caso, ambos no inmigrantes, no se encuentran diferencias. Al buscar el posible origen de esta diferencia, en el sentido de sí venía determinada por la EE de algunos de los padres, hemos visto que en las madres el hecho de ser inmigrante o no, no influye con un $98 \%$ de probabilidad, mientras que en los padres la relación no se observa como significativa. En este sentido, hay más padres no inmigrantes bajos en criticismo que lo esperado y menos altos en dicha escala de $\operatorname{EE}\left(\chi^{2}=3,15 ; p=0,07\right)$. Por otro lado, la frecuencia de padres altos en criticismo es ligeramente superior en los inmigrantes. Siguiendo con los padres, otra de las variables que ha resultado influir en la EE, es la práctica religiosa. En efecto, los padres bajos en EE son más frecuentemente católicos practicantes $\left(\chi^{2}=4,85 ; p=0,02\right)$, y dicha diferencia viene también marcada, por el criticismo. Los padres católicos practicantes presentan bajo criticismo, mientras que los no practicantes son más altos en dicha escala $\left(\chi^{2}=7,09 ; p=0,007\right)$. En el mismo sentido, se ve reflejada la anterior situación en 
ORIGINALES Y REVISIONES

lo referente a hostilidad. De los 23 padres altos en hostilidad, el $65 \%$ son católicos no practicantes $\left(\chi^{2}=5,56 ; p=0,01\right)$. Los estudios, profesión y resto de variables sociodemográficas y familiares no se relacionan con la EE de los padres.

En cuanto a las madres, las que trabajan son, con mayor frecuencia de la esperada, bajas en sobreimplicación emocional, $y$ entre las madres altas en SIE, el $82 \%$ son amas de casa $\left(\chi^{2}=3,01 ; p=0,08\right)$.

\section{Descriptivos}

Los grupos elaborados sobre la base del constructo de EE y el número de sujetos que los componen se describen a continuación:

1-Hogares altos y bajos en EE (EE FAMILIAR). Los puntos de corte utilizados ya se han descrito en el apartado de variables e instrumentos de medida. A continuación se exponen la nomenclatura empleada para los grupos y el número de sujetos que la componen:

EE FAMILIAR (EEFAM):

GRUPO 1: Altos en EE familiar( $\mathrm{N}=53$ familias, $75 \%$ ).

GRUPO 2: Bajos en EE familiar( $\mathrm{N}=17$ familias, $25 \%$ ).

A su vez el grupo 1 de EEFAM se subdivide en dos subgrupos:

GRUPO 1.1: Hogar alto por ambos padres $(\mathrm{N}=26$ familias, $37,1 \%)$.

GRUPO 1.2: Hogar alto por uno de los padres $(\mathrm{N}=27$ familias, $38,6 \%$ ).

EE DE LOS PADRES (EEPA).

GRUPO 1. Altos en EE ( $\mathrm{N}=35$ sujetos, $50 \%$ ).

GRUPO 2. Bajos en $\mathrm{EE}(\mathrm{N}=35$ sujetos, 50\%).
Además, se han clasificado los padres en altos y bajos en las escalas que forman el índice de EE, pero dado que solo hay 8 padres altos en SIE, esta clasificación no se ha utilizado en la presente investigación.

- CRITICISMO DE LOS PADRES (CCPA).

GRUPO 1: Alto en $\mathrm{CC}(\mathrm{N}=33$, $47,1 \%)$.

GRUPO 2: Bajo en $\mathrm{CC}(\mathrm{N}=37$, $52,9 \%$ ).

- HOSTILIDAD PADRES (HPA).

GRUPO 1: Presencia de hostilidad $(\mathrm{N}=26,37,1 \%)$.

GRUPO 2: Ausencia de hostilidad $(\mathrm{N}=44.62,9 \%)$.

Las madres altas en EE se han clasificado en tres grupos, los mismos descritos para el padre, más los grupos de SIE, quedando:

- EE DE LAS MADRES (EEMA).

GRUPO 1. Altas en EE ( $\mathrm{N}=45$, $64,3 \%$ ).

GRUPO 2. Bajas en EE ( $\mathrm{N}=25$, $35,7 \%$ ).

- SIE DE LAS MADRES (SIEMA).

GRUPO 1: Altas en SIE ( $\mathrm{N}=34$, $48,6 \%$ ).

GRUPO 2: Bajas en SIE ( $\mathrm{N}=36$, $51,4 \%)$.

- CRITICISMO DE LAS MADRES (CCMA).

GRUPO 1: Altas en $\mathrm{CC}(\mathrm{N}=31$, $44,3 \%$ ).

GRUPO 2: Bajas en $\mathrm{CC}(\mathrm{N}=39$, $55,7 \%$ ). 
- HOSTILIDAD DE LAS MADRES (HMA).

GRUPO 1: Altas en $\mathrm{H}(\mathrm{N}=24$, 34,35 ).

GRUPO 2: Bajas en $\mathrm{H}(\mathrm{N}=46$, $65,7 \%)$.

Se trata de comprobar la relación entre las escalas de EE del padre y de la madre. Antes de ello exponemos la distribución de estas variables en nuestra muestra (Tabla 1).

-Relación entre las escalas del padre: aparece una esperada alta correlación entre el número de $\mathrm{CC}$ y la hostilidad (rho $=0,77 ; \mathrm{p}=0,000$ ). La SIE y los comentarios positivos no se relacionan con el resto de escalas; mientras que calor mantiene relación negativa con $\mathrm{CC}$ y hostilidad (rho $=-0,42$ para $C C, y$ rho $=-0,49$ para $\mathrm{H}, \mathrm{p}=0,000$ ).

- Relación entre las escalas de la madre: también $\mathrm{CC}$ y $\mathrm{H}$ tienen alta relación (rho $=0,70$ ), pero, curiosamente, la SIE de la madre se asocia a los CC (rho $=0,44$ para $\mathrm{CC} ; \mathrm{p}=0,000$ ). A mayor SIE mayor número de $\mathrm{CC}$, o al revés; $\mathrm{y}$ no en relación a calor o $\mathrm{CP}$, como cabría esperar por estudios anteriores. Se aprecia que a mayor calor expresado por la madre, menor es la hostilidad (rho=-0,46) y menor número de comentarios críticos (rho=0,36). En las madres, calor y CP correlacionan positivamente, si bien de forma moderada $(r=0,34)$.

-Relación entre escalas de padre y madre: la actitud emocional del padre y la madre hacia el paciente tiende a asociarse en los aspectos más negativos de EE, el criticismo y la HOS. El número de CC y la hostilidad que el padre muestra hacia el hijo se correlaciona de forma po- sitiva con el número de $\mathrm{CC}$ y la hostilidad de la madre. Dicho hallazgo se ve reforzado por la correlación negativa con $\mathrm{CP}$, a mayor número de $\mathrm{CC}$ y hostilidad del padre, menor número de $\mathrm{CP}$ de la madre. (Ver Tabla 2).

\section{Relación de EE y variables contextuales}

La relación de las variables sociodemográficas con ambos grupos de variables (contextuales) nos muestra cómo en el padre la familia extensa, entendida como la presencia o ausencia de abuelos, se relaciona con la cantidad de AVE y con la calidad del apoyo social. Los 15 padres que mantienen algún miembro de la familia extensa están sometidos a un mayor número de AVE, frente a los que perdieron a sus padres $(F=3,86 ; p=0,027)$.

Sin embargo, la calidad de las relaciones es mayor en padres sin familia extensa actual, frente a los que sufrieron pérdidas tempranas de padre o madre y frente a los que mantienen la familia $(F=2,72$; $\mathrm{p}=0,073$ ).

Otras dos variables socio-demográficas que se relacionan con las de apoyo/estrés son la inmigración y la religión. En efecto, los católicos practicantes presentan mayor calidad de apoyo social $(t=2,20 ; p=0,03)$ y los no inmigrantes presentan mayor número de $\operatorname{AVE}(\mathrm{t}=2,93$ ; $p=0,005$ ), lo cual se ve reflejado en el nivel de estrés de los padres. Los padres categorizados como alto nivel de estrés son más frecuentemente no inmigrantes, mientras que los bajos en estrés son, en su mayor porcentaje, inmigrantes $\left(\chi^{2}=5,71 ; p=0,02\right)$. La cantidad de apoyo social de los padres no se ve afectada por las características sociodemográficas de la muestra. 
En las madres también resulta significativa la presencia o ausencia de familia extensa en cuanto a la calidad de relaciones sociales, viniendo determinada por una peor calidad de apoyo en las madres que sufrieron pérdidas tempranas de sus padres, frente a los otros dos grupos $(\mathrm{F}=4,30$ ; $\mathrm{p}=0,02)$. Asimismo, la calidad de apoyo se relaciona en las madres con la religión. De este modo, las católicas practicantes tienen mejor calidad de apoyo $(t=2,42$; $p=0,02$ ). La inmigración, en las madres, determina la cantidad de apoyo, en el sentido de que las no inmigrantes tienen más apoyo $(\mathrm{t}=1,95 ; \mathrm{p}=0,05)$, pero $\sin$ influir en la calidad de éste.

En las madres que no trabajan fuera de casa aparece la tendencia $(p=0,07)$ a un menor apoyo social y a un mayor número de AVE. De hecho, todas las madres con alto nivel de estrés son amas de casa.

En cuanto a nivel de estrés, una mayoría de sujetos (padre y madre) tienen bajo número de AVE y un apoyo moderado. Tenemos un $90 \%$ de madres con bajo número de $\mathrm{AVE}$ y un $88 \%$ con apoyo moderado o alto. En los padres el $90 \%$ tienen apoyo moderado o alto y un $55 \%$ de ellos, presentan niveles de estrés bajos. En la Tabla 3 se pueden comprobar las medias y desviaciones estándar de estas variables.

El estudio de las relaciones entre las variables muestran que la calidad y cantidad de apoyo no están relacionadas y son, asimismo, independientes apoyo y AVE. Sin embargo, el apoyo del padre y de la madre presentan correlación positiva y significativa $(r=0,34 ; p=0,005)$ al igual que el número de AVE ( $\mathrm{r}=0,34$; $\mathrm{p}=0,007$ ).

\subsection{Hogares altos y bajos en EE.}

No muestran diferencia alguna en las escalas de apoyo ni en cuanto a acontecimientos vitales estresantes.

\subsection{Padres altos y bajos en EE.}

Se ha estudiado si el apoyo social de los padres y los AVE pueden estar relacionados con la EE del padre. Observamos que a pesar de que el grupo alto en EE tiene mayor número de AVE y menor apoyo social, en cantidad y calidad, que los padres bajos en EE, estas diferencias no son significativas al nivel 0,05 . No obstante, como se acerca a 0,10 se ha comprobado si los altos y bajos en criticismo o SIE se diferencian entre sí.

En este caso, el apoyo social en calidad ( $p=0,07$ ) pero sobre todo en cantidad permite diferenciar a los padres altos en criticismo de los bajos. Se observa que los padres altos en criticismo tienen menor apoyo social ( $\mathrm{p}=0,04)$, mientras que la SIE no presenta relación alguna con la red social. La relación entre ambas variables, aunque moderada $(0,27)$, indica, en el mismo sentido, que a mayor número de $\mathrm{CC}$ menor apoyo social y viceversa. Aunque el criticismo tiene relación con la religión, en los padres el apoyo social no parece estar influenciado por la religión con lo cual concluimos que criticismo y cantidad de apoyo social son dos variables no independientes.

Sin embargo, la calidad de apoyo social puede decirse que viene mediada por la religión. Los padres altos en criticismo son católicos no practicantes más frecuentemente, mientras que los padres católicos no practicantes tienen también menor calidad de apoyo, por lo que el he 
cho de que los padres altos en criticismo presenten menor calidad de apoyo puede ser debido a un sesgo radicado en la variable religión

\subsection{Madres altas y bajas en EE.}

No se observan diferencias en apoyo ni AVE entre estos dos grupos. No obstante, entre las madres altas y bajas en criticismo se comprueba, como en el caso de los padres, una menor calidad de apoyo social en las madres críticas $(t=-2,32$; $\mathrm{p}=0,02$ ). No se observa relación con las variables sociodemográficas de la madre y el criticismo de la madre no está influido, tampoco, por las características de la muestra, con lo cual concluimos que las madres con 6 ó más comentarios críticos tienen una peor calidad de apoyo social. La relación entre el número de $\mathrm{CC}$ y la calidad de apoyo no es significativa $(r=-$ 0,16 ). Los $\mathrm{CP}$ presentan relación positiva con la cantidad de apoyo (rho $=0,21)$ y su calidad ( $r h o=0,25 ; p=0,05)$ si bien de forma no significativa.

\section{Discusión}

Nuestra muestra es la segunda más amplia en familiares a los que se ha pasado la CFI (140) y la única en el que en el $100 \%$ de los pacientes se ha evaluado a ambos padres. Además hay que señalar que los pacientes seleccionados cumplían criterios restrictivos de esquizofrenia (DSM-III-R), edad limitada (15-35) y eran solteros. Contamos con un $75 \%$ de hogares altos en EE, el más alto porcentaje publicado hasta la fecha. Si comparamos padres y madres por separado, tenemos un $50 \%$ de padres altos en EE y un 64,3\% de madres, lo que colocaría a nuestros resultados en un lugar intermedio en cuanto a frecuencia de familiares altos en EE, estando más cerca de las muestras británicas los porcentajes de nuestras madres y de las angloamericanas los padres. De lo cual se deduce que si no hubiéramos incluido a ambos padres no habríamos tenido un porcentaje tan elevado de hogares altos en EE.

En cuanto al criticismo de padres (45\%) y madres $(44,3 \%)$, nuestra muestra se sitúa en un rango medio. En hostilidad, sin embargo, somos los que más familiares hostiles encontramos ( $37 \%$ para padres y $34,3 \%$ para madres). Solo hay 8 padres altos en SIE $(11,8 \%)$ cifra similar a la de otros autores y un $48,6 \%$ de madres altas en SIE, que coloca a nuestra muestra también en el más alto rango en esta escala.

Las relaciones de las escalas de EE entre padre y madre muestran cómo ambos coinciden en los aspectos más negativos (CC y HOS), pero no en SIE, lo cual indica que cuando medimos solo a un miembro de la familia conseguimos recoger el criticismo familiar pero no la sobreimplicación, lo cual debería ser tenido en cuenta en posteriores estudios. Las correlaciones halladas entre las escalas hablan a favor de la validez del constructo.

El que la alta EE se asocie a que ambos padres sean inmigrantes puede ser debido a la falta de apoyo de la familia de origen y el hecho de que los padres no inmigrantes sean menos críticos puede ser 
debido a aspectos culturales, entre los que sugerimos que quizás sean menos críticos, o más ausentes, o que tengan más apoyo de su familia de origen.

El que los padres católicos practicantes sean más bajos en criticismo y $\mathrm{H}$, puede ser debido a que tienen unos valores religiosos y/o éticos que les ayuda a tener una actitud más tolerante hacía la enfermedad, quizás dándole un sentido trascendente, por ejemplo, como prueba divina. Es posible también que a través de las prácticas religiosas obtengan un mayor soporte social. Los padres bajos en EE, como ya hemos expuesto, son más frecuentemente católicos practicantes, el hallazgo de que estos tienen mayor calidad de apoyo social, implica que ambos factores conllevan una actitud más positiva hacia los hijos.

Como ya señalaron Brown et al (1), encontramos que la SIE es menor en las madres que trabajan. Ello puede ser debido a que al ejercer un rol más instrumental tengan una respuesta emocional más baja, a que tenga intereses que le permitan su propio desarrollo y no necesite implicarse en una relación simbiótica con el hijo y, finalmente, a que el tiempo y la dedicación al trabajo le impida ejercer un control excesivo sobre el hijo.

En padres de esquizofrénicos hemos encontrado una buena calidad de apoyo $(84,4 \%$ de media en los padres y $85,1 \%$ en las madres) y un nivel de apoyo moderado en ambos que, salvo en casos de estrés (tanto en padres como en madres) pudiera ser suficiente. Esto contrasta con los resultados de Brown et al. (3), Hammer
(42) Goldman (44), Platt (43) quienes hallaron que los padres de esquizofrénicos tenían baja red social de apoyo y aislamiento social. Estos resultados pueden deberse a que realmente hay diferencias entre los familiares anglosajones y españoles o a los instrumentos de medida utilizados. De todas formas, el apoyo social moderado es insuficiente para afrontar el estrés crónico que produce el tener en el hogar un hijo esquizofrénico, el $45 \%$ de los padres tenían estrés alto.

$\mathrm{El}$ hecho de que los padres y madres altos en criticismo tengan menos apoyo social, puede estar relacionado con la carga familiar y el estigma social. En las madres el mayor apoyo social se asocia a más $\mathrm{CP}$, lo cual puede deberse a que pueden eliminar tensión en su red social, sentirse comprendida y, desde ahí, comprender y valorar más al hijo.

Los familiares altos en criticismo tienen menos cantidad de apoyo social que los bajos en CC, y las madres, a mayor número de comentarios críticos, peor calidad de apoyo. Es posible que la falta de apoyo en el que descargar sus tensiones generadas en la relación con el hijo dé lugar a un mayor criticismo, aunque también podría interpretarse que si su criticismo se extiende a sus relaciones sociales estas últimas sean de menor calidad. Estos datos nos hacen pensar que el criticismo puede deberse a una falta de habilidades sociales que les hace, o bien retraerse (baja red social), o bien mantener relaciones conflictivas con el entorno (mala calidad), datos que matizan los hallazgos encontrados por los autores ante 
riormente citados en cuanto a la baja red social de los padres de esquizofrénicos. El nivel de estrés provocado por acontecimientos vitales (el estrés familiar hace referencia a pérdidas o incorporación de nuevos miembros a la familia, enfermedades de familiares y cambios de residencia) en el último año es moderado en padres y bajo en madres; esto supone una acumulación de factores que aumentan la carga familiar. Además, hemos hallado en nuestro estudio que los familiares bajos en EE y las madres bajas en criticismo puntúan más alto en estrés familiar que los altos. Parece ser que la aparición de estos acontecimientos estresantes familiares hacen que los padres centren su atención en esos problemas y no en el hijo. En cuanto a los padres, destaca que cuanto más bajos son en SIE más estrés laboral tienen, posiblemente porque la excesiva preocupación por los problemas de trabajo no les permita sobreimplicarse con el hijo.

En los padres, la existencia de familia extensa se asocia a más AVE, lo cual es normal pues hay más personas cercanas a las que les suceden cosas que pueden ser estresantes y el no tenerla se asocia a más calidad de apoyo, quizás debido a que el hecho de no tener familia extensa les lleve a buscar afecto y apoyo en otras relaciones mejorando su calidad. En las madres, el que la calidad de apoyo sea peor cuando han perdido a alguno se sus padres en la infancia puede tener que ver con duelos no elaborados y con déficits en las relaciones familiares, quizá estilos de apego deficientes que han dificul- tando la socialización y problemas típicos de las familias monoparentales (52). Los no inmigrantes tienen más cantidad de apoyo, lo cual puede estar asociado a la existencia de familia extensa.

Las amas de casa tienen más estrés, menos apoyo y más AVE y son más altas en SIE posiblemente debido a que están aisladas, con problemas y buscan el apoyo en el paciente.

Todos estos datos nos orientan hacia intervenciones del tipo de grupo de apoyo de familiares. En este sentido, las intervenciones de Leff et al. (19) que incluían grupo de apoyo de familiares y charlas psicopedagógicas, consiguieron reducir el criticismo y la tasa de recaídas. En un estudio realizado por nosotros también conseguimos reducir la tasa de recaídas con grupos de familiares $(53,54)$. No podemos saber si la reducción de CC se debió al apoyo que brindaba el grupo, o a la mejor comprensión de la enfermedad debido a las charlas psicopedagógicas, como señalaron Leff et al. (19). Nuestros resultados sugieren que las intervenciones que favorecen el apoyo social de familiares de esquizofrénicos deben tenerse muy en cuenta a la hora de diseñar programas tendentes a reducir la tasa de recaídas y a mejorar la calidad de vida de pacientes y familiares. Por otro lado, sería interesante estudiar mejor el efecto de ambos elementos de la intervención por separado para ver cual de los dos, psicopedagogía o apoyo del grupo, son más eficaces para reducir la EE. 
ORIGINALES Y REVISIONES

TABLA I. Distribución de las puntuaciones en las escalas de EE, de padres y madres.

\begin{tabular}{|c|c|c|}
\hline ESCALAS & PADRES & MADRES \\
\hline CC (Media \pm S.D. $)$ & $6,44 \pm 5,05$ & $6,21 \pm 4,42$ \\
\hline CP (Media \pm S.D.) & $1,07 \pm 1,34$ & $1,55 \pm 1,50$ \\
\hline $\begin{array}{l}\text { SIE } \\
\qquad \begin{array}{r}\mathrm{N} \\
\%\end{array}\end{array}$ & $\begin{array}{llllll}\mathbf{0} & \mathbf{1} & \mathbf{2} & \mathbf{3} & \mathbf{4} & \mathbf{5} \\
12 & 21 & 29 & 7 & 1 & - \\
17 & 30 & 41 & 10 & 1 & -\end{array}$ & $\begin{array}{cccccc}\mathbf{0} & \mathbf{1} & \mathbf{2} & \mathbf{3} & \mathbf{4} & \mathbf{5} \\
- & 7 & 29 & 18 & 15 & 1 \\
- & 10 & 41 & 26 & 21 & 1\end{array}$ \\
\hline $\begin{array}{l}\text { HOSTILIDAD } \\
\text { N } \\
\%\end{array}$ & $\begin{array}{llll}\mathbf{0} & \mathbf{1} & \mathbf{2} & \mathbf{3} \\
44 & 13 & 2 & 11 \\
63 & 18 & 3 & 15\end{array}$ & $\begin{array}{llcc}\mathbf{0} & 1 & 2 & 3 \\
46 & 10 & 5 & 9 \\
66 & 14 & 7 & 13\end{array}$ \\
\hline $\begin{array}{c}\text { CALOR } \\
\mathrm{N} \\
\%\end{array}$ & $\begin{array}{rrrrrr}\mathbf{0} & \mathbf{1} & \mathbf{2} & \mathbf{3} & \mathbf{4} & \mathbf{5} \\
1 & 11 & 27 & 22 & 8 & 1 \\
1 & 16 & 39 & 31 & 11 & 1\end{array}$ & 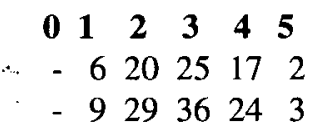 \\
\hline
\end{tabular}

TABLA 2. :Matriz de correlaciones entre escalas de EE, de padres y madres.

\begin{tabular}{|l|c|c|c|c|c|c|}
\hline & MADRE & SIE & CC & HOS & CP & CALOR \\
\hline PADRE & & & & & & \\
SIE & & 0,20 & 0,26 & 0,24 & 0,00 & $-0,10$ \\
CC & & 0,07 & $0,45^{*}$ & $0,37^{*}$ & $-0,26$ & $-0,11$ \\
HOS & & 0,17 & $0,40^{*}$ & $0,38^{*}$ & $-0.33 *$ & $-0,09$ \\
CP & & 0,03 & $-0,06$ & $-0,21$ & 0,00 & 0,06 \\
CALOR & & 0,09 & $-0,10$ & $-0,08$ & 0,15 & 0,02 \\
\hline
\end{tabular}

$* p=0,005$

TABLA 3. Distribución (Media y S.D.) de las variables de Apoyo y AVE, para Padre, Madre, y Familia.

\begin{tabular}{|l|c|c|c|c|c|c|}
\cline { 2 - 7 } \multicolumn{1}{c|}{} & \multicolumn{2}{c|}{ PADRE } & \multicolumn{2}{c|}{ MADRE } & \multicolumn{2}{c|}{ NIVEL FAMILIAR } \\
\cline { 2 - 8 } \multicolumn{1}{c|}{} & MEDIA & S.D. & MEDIA & S.D. & MEDIA & S.D. \\
\hline $\begin{array}{l}\text { CANTIDAD } \\
\text { APOYO }\end{array}$ & 23,18 & 5,1 & 23,33 & 5,7 & 23,25 & 5,4 \\
\hline $\begin{array}{l}\text { CALIDAD } \\
\text { APOYO }\end{array}$ & 84,44 & 6,0 & 85,11 & 8,4 & - & - \\
\hline AVE & 9,73 & 7,2 & 7,28 & 6,2 & 13,33 & 8,2 \\
\hline
\end{tabular}




\section{BIBLIOGRAFIA}

1. Brown, G.W., Monck, E.M., Carstairs, G.M. \& Wing,J.K., (1962). "Influence of family life on the course of schizophrenic illness". Brit. J. Preventive and Soc. Med., 16, 55-68.

2. Brown, G.W. \& Rutter, M., (1966). "The measurement of family activities and relationships. A methodological study", Human Relations, 19: 241-263.

3. Brown, G.W., Birley, J.L.T. \& Wing, J.K., (1972). "Influence of family life on the course of schizophrenic disorders: a replication". Brit. J. Psychiat., 121, 241-258.

4. Vaughn, C. \& Leff, J., (1976a). "The influence of family and social factors on the course of psychiatric illness: a comparison of schizophrenic and depressed neurotic patients", Brit. J. Psychiat., 129, 125-137.

5. Hooley, J.M., (1986). "Expressed emotion and depression: interactions between patients and High-EE versus Low-EE spouses", J. Abn. Psychol., 95, 3, 237-246.

6. Vaughn, C., Snyder, K.S., Jones, S., Freeman, W.B. \& Falloon, I.R.H., (1984). "Family factors in schizophrenia relapse. Replication in California of British research

7. Moline, R.A., Singh, S., Morris, A., \& Meltzer, H., (1985). "Family expressed emotion and relapse in schizophrenia in 24 urban american patients", Am. J. Psychiat., 142, 1078-1081.

8. Karno, M., Jenkins, J., Selva, A., Santana, F., Telles, C., Lopez,S. \& Mintz, J., (1987). "Expressed emotion and schizophrenia outcome among Mexican-American families", The J. of Nervous and Mental Desease, 175, 143-151.

9. Leff, J. (1985). "Social factors and maintenance neuroleptics in schizophrenic relapse: An integrative mode"'. Integrative Psychiatry, 3, 72-82.

10. Leff, J. \& Vaughn, C., (1985). Expressed emotion in families. Its significance for mental illness, New York: The Guilford Press.
11. Leff, J., Wig, N., Ghosh, A., Beedi, H., Menon, D., Kuipers, L., Korten, A., Ernberg, G., Day, R., Sartorius, N. \& Jablensky, A., (1987). "Influence of relatives' expressed emotion on the course of schizophrenia in Chandigarh", Brit. $J$. Pychiat., 151, 166-73.

12. Pellizzer, G., Barrelet, L. \& Ammann, E., (1987). «'Emotion exprimée' par la famille et évolution d'un group de patients schizophrènes en milieu francophone", Revue Suisse de Psychologie, 46 (1/2), 33-40.

13. Gutiérrez, E., Escudero, V., Valero, J.A. et al., (1988). "Expresión de emociones y curso de la esquizofrenia: II. Expresión de emociones y curso de la esquizofrenia en pacientes en remisión", Análisis y Modificación de Conducta, 15, 275-316.

14. Arevalo, J. y Vizcarro, C., (1989). "Niveles de 'Emoción Expresada' en familiares de pacientes esquizofrénicos. Datos para una comparación transcultural", Rev de la Asoc. Esp. de Neuropsiq., 9, 437-450..

15. Cazzullo, C.L., Bressi, C., bertrando, P., Clerici, M. \& Maffei,C., (1989). "Schizophrenie et expression emotionnelle familiale. Etude d'une population italienne". L'Encéphale, 15, 1-6.

16. Santos, A., (1995). "Factores familiares (Emoción Expresada) y su incidencia en la tasa de recaídas en paciente esquizofrénicos". Tesis Doctoral, no publicada.

17. Bebbington, P. y Kuipers, L. (1994). "The predictive utility of expressed emotion in schizophrenia: an aggregate analysis", Psychological Medicine, 24, 707-718.

18. Goldstein, M.J. (Ed). (1981). New directions for mental health services: new developments in intervention with families of schizophrenics. S. Francisco: Jossey-Bass.

19. Leff, J., Kuipers, L., Berkowitz, R., Eberlein-vries, R. \& sturgeon, D., (1982). "A controlled trial social intervention in the families of schizophrenic patients", Brit. J. Psychiat., 141, 121-134. 
20. Leff, J.(1994), "Working with the families of schizophrenic patients" Brit. J. Psychiat., 164, (Supp. 23), 71-76.

21. Falloon, I.R. \& Liberman, R.P.,(1983). "Interactions between drug and psychosocial therapy in schizophrenia", Schizophr. Bull., 9, 4, 543-554.

22. Anderson, C., Reiss, D. \& Hogarty, g., (1986). Schizophrenia and familiy. New york. The Guilford Press.

23. Cozolino, J.L., Goldstein, M.J., Nuechterlein, K.H., West, K.L. \& Snyder, K.S., (1988). "The impact of education about schizophrenia on relatives varying in expressed emotion", Schizophr. Bull., 14, 4, 675-687.

24. Tarrier, N., Barrowclough, C., Vaughn, C., Bamrah, J.S., Porceddu, K., Watts, S. \& Freeman, H., (1989). "Community management of schizophrenia. A two-year follow-up of a behavioural intervention with families", Brit. J. Psychiat., 154, 625-628.

25. Tarrier, N. \& Barrowclough, C., (1995). "Family interventions in schizophrenia and their long-term outcomes", Int. J. Ment. Health, vol. 24, $\mathrm{n}^{\circ} 3,38-53$.

26. Barrowclough, C. \& Tarrier, N., (1990). "Social functioning in schizophrenic patients. I. The effects of expressed emotion and family intervention". Soc. Psychiat. Psychiatric Epidemiology, 25: 125-129,

27. Mozny, P. \& Votypkova, P., (1992). "Expressed Emotion, relapse rate and utilization of psychiatric inpatient care in schizophrenia. A study from Czechoslovakia", Soc. Psychiat. and Psychiatric Epidemiology, 27, 174-179.

28. Hogarty, G.E., (1993). "Prevention of relapse in chronic schizophrenic patients", J. Clin. Psychiat., 54:3 (supp), March, 18-29.

29. Xiang, M., Ran, M., Li, S., (1994). “A controlled evaluation of psychoeducational family intervention in a rural Chinese community", Brit. J. Psychiat., 165, 544-546.

30. Goldstein, M.J., (1994). "Psychoeducational and family therapy in relapse prevention". Acta Psychiatrica Scandinavica, 89 (Supp. 382), 54-57.
31. Goldstein, M.J., (1995). "Psycoeducation and relapse prevention", Int. Clin. Psychopharma, 9 suppl. 5, 59-69.

32. Leff, J., Berkowitz, R., Shavit, N., Strachan, A., Glass, I. \& Vaughn, C., (1989). "A trial of family therapy v. a relatives group for schizophrenia", Brit. $J$. Psychiat., 154, 58-66.

33. Tarrier, N., Barrowclough, C., Vaughn, C., Bamrah, J.S., Porceddu, K., Watts, S. \& Freeman, H., (1988). "The community management of schizophrenia. A controlled trial of a behavioural intervention with families to reduce relapse", Brit. J. Psychiat., 153, 532-542.

34. Vaughn, C. \& Leff, J., (1976b). "The measurement of expressed emotion in the families of psychiatric patients", Brit. $J$. Soc. Clin. Psychol., 15, 157-165.

35. Wing, N.N., Menon, D.K., Bedi, H., Ghosh, A., Kuipers, L., Leff, J., Korten, A., Day, R., Sartorius, N. y cols. (1987). "Expressed emotion and schizophrenia in North India. I. Cross-cultural transfer of ratings of relatives' expressed emotion". British Journal of Psychiatry, 151, 156-173.

36. Parker, G. y Johnson, P. (1987). "Parenting and schizophrenia: an Australian study of expressed emotion". Australian and New Zealandan Journal of Psychiatry, 21, 60-66.

37. Bertrando, P., Beltz, J., Bressi, C., Clerici, M., Farma, T., Invernizzi, G., Cazullo, C.L. (1992). "Expressed emotion and schizo-phrenia in Italy. A study of an urban population". British Journal of Psychiatry, 161, 223-229.

38. Barrelet, Von L., Pellizzer, G., Ammann, L. (1988). "Family expressed emotion and outcome of schizophrenics: A study in a french cultural environment". Archives Suisses de Neurologie et de Psychiatrie, $139(5), 27-34$.

39. Vizcarro, C. y Arévalo, J. (1986). "Emoción Expresada: Introducción al concepto, evaluación e implicaciones pronósticas y terapéuticas". Estudios de Psicología, 27-28, 89-109. 
40. Cannon-Spoor, H.E., Potkin, S.G., Wyatt, R.J. (1982). "Measurement of premorbid adjustment in chronic schizophrenia". Schizophrenia Bulletin, 8(3), 470-484.

41. Montero, I., Gómez-Beneyto, M., Ruiz, I., Puche, E., Adam, A. (1992). "The influence of family expressed emotion on the course of schizophrenia in a sample of spanish patients. A two-year follow-up study". British Journal of Psychiatry, 161, 217-222.

42. Hammer, M., (1981). "Social support, social netwoks, and schizophrenia", Schizophr. Bull., 1, 45-47.

43. Platt, S., (1985) "Measuring the burden of psychiatric illness on the family: an evaluation of some rating scales", Psychol. Med., 15, 382-394.

44. Goldmann, H.H., (1982). "Mental illness and family burden: a public health perspective". Hosp. and Community Psychiat., 33, 7, 557-660.

45. Fadden, G., BebbingtoN, P. \& Kuipers, L., (1987). "The burden of care: the impact of functional psychiatric illness on the patient family", Brit. J. Psychiat., 150, 285-292.

46. Carpentier, N., Lesage, A., Goulet, J., Lalonde, P. \& Renaud, M., (1992). "Burden of care of families not living with young schizophrenic relatives". Hosp. and Community Psychiat., vol. 43, nº1, 38-43

47. Anderson, C., Hogarty, G., Bayer, T., Needleman, R., (1984). "Expressed emotion and social networks of parents of schizophrenic patients", Brit. J. Psychiat, 144: $247-255$.
48. Bentsen, H., Boye, B., Munkvold, O. et al. (1996). "Emotional Overinvolvement in parents of patients with schizophrenia or related psychosis: demographic and clinical predictors". British Journal of Psychiatry, 169, 622-630.

49. Barrowclough, C., Tarrier, N. \& Johnston, M. (1996) «Distres, Expressed Emotion and attributions on relatives of schizophrenia patients» Schizophrenia Bulletin, 22 (4) 691-701.

50. Conde, V. y Franch, J. I., (1984). "Escalas de evaluación comportamental para la cuantificación de la sintomatología psicopatológica en los trastornos ansiosos y depresivos", Valladolid: Departamento de Psicología Médica del Hospital Clínico de la Facultad de Medicina de Valladolid.

51. Fisher, L., Kokes, R.F., Ransom, D.C., Phillips, S.L., Rudd, P. (1985). "Alternative strategies for creating «relational» family data", Family Process, 24, 213-224.

52. Espina, A. (1995). "Familias mono-parentales y reconstituídas”. En A. Espina, B. Pumar y M. Garrido. Problemáticas familiares actuales y terapia familiar. Valencia. Promolibro.

53. Espina, A., Pumar B., Santos, A. y Ayerbe, A. (1996a). "Intervenciones familiares para reducir la tasa de recaídas en la esquizofrenia". En A. Espina y B. Pumar. (Eds.) Terapia familiar sistémica. Teoría, clínica e investigación. Madrid. Fundamentos.

54. Espina, A., Pumar B., Santos, A. y Ayerbe, A. (1996b). "Terapia familiar en la esquizofrenia". En A. Espina y B. Pumar. (Eds.) Terapia familiar sistémica. Teoría, clínica e investigación. Madrid. Fundamentos.

* Psiquiatra. Profesor titular y Director del Master de Terapia familiar y de Pareja del Universidad del País Vasco.

** Médico. Psicóloga. Departamento de Personalidad, Evaluación y Tratamientos Psicológicos. Facultad de Psicología.

Universidad del País Vasco.

Fecha de recepción: 14-IX-1999

Correspondencia: A. Espina. Facultad de Psicología. Avda. de Tolosa, 70 - 20009-SAN SEBASTIÁN 